\title{
CARACTERÍSTICAS DO APOIO SOCIAL ASSOCIADOS À PREMATURIDADE EM UMA POPULAÇÃO DE PUÉRPERAS DE BAIXA RENDA
}

\author{
Characteristics of social support associated with prematurity in a population of mothers \\ of low income \\ Características del apoyo social asociadas con la prematuridad en una población de \\ madres de baj os ingresos.
}

Elaine da Costa Guimarães ${ }^{1}$

Enirtes Caetano Prates Melo²

\begin{abstract}
RESUMO
Trata-se de um estudo caso-controle que teve como objetivo investigar a associação entre prematuridade e o nível de apoio social, segundo variáveis socioeconômicas, demográficas e relacionadas à saúde e à assistência, em uma população de puérperas de baixa renda atendidas em um hospital público do município do Rio de Janeiro. Foram entrevistados 108 casos e 228 controles, em um total 336 mulheres. A idade das mulheres variou entre 14 e 45 anos. A média da idade foi de 25,8 anos (desvio-padrão: $6,9)$ para os casos e de 24,9 (desvio-padrão 6,5) para os controles. Quanto à raça/etnia autorreferida, 77,1\% denominaramse não brancas. Da amostra, 68,8\% iniciaram o pré-natal antes do terceiro trimestre gestacional. 0 número de consultas durante o pré-natal, ocorrência de ameaça de parto prematuro e história de prematuridade anterior mostraram-se associados ao nível de apoio, calculado a partir do escore total das dimensões do apoio social.
\end{abstract}

Palavras-chave: Apoio Social. Nascimento Prematuro. Saúde Materno-infantil.

\begin{abstract}
This is a case-control study aimed to investigate the association between preterm birth and level of social support, according to socioeconomic, demographic and health-related assistance and, in a population of low-income mothers attending a public hospital the city of Rio de Janeiro. We interviewed 108 cases and 228 controls, totaling 336 women. The women's ages ranged from 14 to 45 years. The mean age was 25.8 years (standard deviation: 6.9) for cases and 24.9 (standard deviation 6.5) for controls. Regarding race/ethnicity self-reported $77.1 \%$ called themselves as nonwhite. Women, $68.8 \%$ began prenatal care before the third quarter. The number of visits during the prenatal period, occurrence of threatened preterm delivery and previous history of prematurity were associated with the level of support, calculated from the total score of the dimensions of social support.
\end{abstract}

Keywords: Social support. Premature birth. Maternal and child health.

\section{Resumen}

Se trata de un estudio caso-control cuyo objetivo fue investigar la asociación entre el nacimiento prematuro y el nivel de apoyo social, según indicadores socioeconómicos, demográficos y de asistencia relacionados con la salud y, en una población de madres de bajos ingresos que asisten a un hospital público en la ciudad de Río de Janeiro. Fueron analizados 108 casos y 228 controles, de un total de 336 mujeres. Las edades de las mujeres oscilaron entre 14 y 45 años. La edad media fue de 25,8 años (desviación estándar: 6,9) para los casos y el 24,9 (desviación estándar de 6,5) para los controles. En cuanto a la raza/origen étnico auto-reportado $77,1 \%$ se denominaron no blancos. El 68,8\% comenzó la atención prenatal antes del tercer trimestre de embarazo. El número de visitas durante el período prenatal, ocurrencia de amenaza de parto prematuro y antecedentes de prematuridad se asociaron con el nivel de apoyo, se calcularon a partir de la puntuación total de las dimensiones de apoyo social.

Descriptores: Apoyo social. Nacimiento prematuro. Salud materno-infantil.

${ }^{1}$ Enfermeira, Mestranda do Programa de Pós-Graduação - Mestrado em Enfermagem da Universidade Federal do Estado do Rio de Janeiro - UNIRIO.

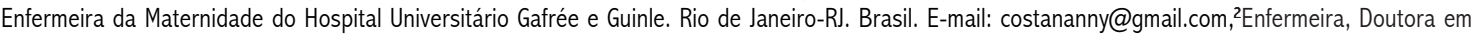
Ciências pela Escola Nacional de Saúde Pública - FIOCRUZ. Professora Adjunta do Departamento de Enfermagem em Saúde Pública da Escola de Enfermagem Alfredo Pinto da Universidade Federal do Estado do Rio de Janeiro-RJ. Rio de Janeiro. Brasil. E-mail: enirtes@globo.com 


\section{INTRODUÇ̃̃O}

A prematuridade envolve $5 \%$ a $10 \%$ das gestações e representa um dos fatores determinantes na ocorrência do óbito infantil. ${ }^{1}$ No Brasil, a taxa de prevalência de prematuridade é de aproximadamente $7 \%$, com uma variação em relação às diferentes regiões e capitais. Dentre os mecanismos explicativos para a prematuridade encontram-se fatores de natureza biológica, social e relacionados à assistência à saúde. 0 tipo de parto; a raça/etnia, a idade, o estado civil, a ocupação, o estado nutricional da mãe; o tabagismo; a história reprodutiva materna (prematuridade anterior, cirurgia ginecológica prévia); condições da gestação (gestação gemelar, sangramento durante a primeira metade da gestação, piolonefrite, vaginose bacteriana e infecção urinária durante a gestação); a exposição a substâncias tóxicas, a qualidade do pré-natal representam fatores destacados na literatura. ${ }^{2,3}$

A ausência de uma rede de apoio pode influenciar 0 desencadeamento de desfechos como prematuridade e baixo peso, a partir de sua associação com fatores biológicos, psíquicos e neuroendócrinos. Em momentos de grande fragilidade, como a gravidez, em que muitas transformações físicas e emocionais ocorrem no corpo e na mente da mulher; 0 apoio social pode representar um recurso inestimável. Nos últimos anos, um número crescente de estudos vem demonstrando que as interações sociais têm um impacto positivo sobre a saúde física e o bem-estar psicológico. ${ }^{4-9} \mathrm{~A}$ partir da década de 70 , alguns autores se dedicaram a pesquisas voltadas para a compreensão dos mecanismos pelos quais o apoio social pode prevenir, ou atenuar, as consequências negativas relacionadas a eventos de crise ou transição ao longo da vida. ${ }^{5}$

0 apoio social tem sido amplamente discutido nos países desenvolvidos, sendo apresentado como um importante fator que contribui na redução do estresse e no desenvolvimento de mecanismos de enfrentamento, ajustamento e adaptação nas diferentes situações vivenciadas pelos indivíduos.?

0 apoio social pode ser definido como um sistema de relações interpessoais com ênfase na disponibilidade de ajuda em situações de necessidade. Pode funcionar como um mediador (efeito buffer), protegendo e amenizando situaç̃̃es causadoras de estresse no indivíduo, como através de um efeito direto em que os recursos sociais atuariam "tamponando" a resposta do organismo ao processo de desenvolvimento de desfechos desfavoráveis. ${ }^{8}$

Atreladas ao conceito de apoio estão as redes sociais que correspondem ao aspecto estrutural, caracterizado por um conjunto de pessoas que estabelecem interações entre si, de onde emergem relações de apoio entre seus integrantes. Desde o nascimento até a morte nos encontramos envolvidos por uma cadeia de relacionamentos interpessoais, que a princípio é constituída pela família, amigos, colegas de estudo e trabalho que estimulam a construção das relações sociais, culturais, religiosas, esportivas e de cuidados com a saúde, gerando práticas sociais. ${ }^{6,8}$

Pesquisas têm revelado que fatores sociodemográficos, biológicos e hábitos de vida respondem apenas por $30 \%$ dos nascimentos prematuros, mostrando que existem outros fatores que estão envolvidos no complexo causal, dentre os quais os fatores psicossociais. ${ }^{9}$ Gestantes com maior disponibilidade de apoio teriam comportamentos mais saudáveis com abandono de práticas prejudiciais à saúde, maior adesão ao pré-natal, incluindo o uso de serviços de saúde preventiva que auxiliam na detecção precoce de problemas relacionados à gestação. Estudo realizado com gestantes no município do Rio de Janeiro mostrou que mulheres multíparas, com maior nível de apoio, tiveram um risco menor para ocorrência da pré-eclampsia. ${ }^{10}$

Muito embora a literatura tenha demonstrado o efeito benéfico proporcionado pelo apoio social, estudos adicionais que se atenham à influência dos recursos sociais sobre a saúde vêm contribuir para a compreensão dessa complexa rede. 0 presente estudo tem por objetivo investigar a associação entre prematuridade e o nível de apoio social, segundo variáveis socioeconômicas, demográficas e relacionadas à saúde e à assistência em um hospital da rede pública do município do Rio de Janeiro.

\section{MATERIAIS E MÉTODOS}

Trata-se de um estudo prospectivo com abordagem quantitativa, com um desenho caso-controle. A pesquisa foi realizada em um hospital maternidade da rede pública do Município do Rio de Janeiro, que atende gestantes de risco para complicações obstétricas. Selecionaram-se como população elegível para o estudo puérperas que se encontravam no período de pós-parto mediato, no período de janeiro a abril de 2009.

Foram considerados casos todas as puérperas cujos filhos nasceram prematuramente, entre 22 a 36 semanas e 6 dias, durante o período de desenvolvimento do estudo. Como controles foram recrutadas mulheres cujos recém-nascidos nasceram a termo (idade gestacional maior ou igual a 37 semanas). 0 grupo de controles hospitalares foi formado por uma amostra aleatória de puérperas internadas cujo parto foi a termo. A seleção aleatória dos controles levou em conta a ordem de nascimento do recém-nascido; buscou-se selecionar aqueles que nasceram no mesmo dia e em horário próximo ao nascimento do recém-nascido, definido como caso a partir dos critérios estabelecidos. Para o processo de identificação dos casos e seleção aleatória dos controles foi realizado um monitoramento diário do Livro de Registro da sala de parto. As puérperas foram selecionadas em uma proporção de dois controles para um caso (2:1), sendo então entrevistados 108 casos e 228 controles.

A fim de estabelecer a idade gestacional, adotou-se a data da última menstruação (DUM) relatada pela mãe no momento da entrevista, concordante com a data da última 
menstruação e a ultrassonografia registradas no prontuário obstétrico.

0 instrumento de coleta de dados foi composto por duas partes. A primeira foi composta por identificação materna, local e condições de moradia, escolaridade, renda familiar, atividades laborais, antecedentes obstétricos, informações sobre a gestação atual e assistência pré-natal. A segunda parte correspondeu ao bloco de apoio social que abrange perguntas relacionadas à rede social e ao apoio social. Para avaliar 0 apoio social foi utilizada a versão para a língua portuguesa da escala produzida para o Medical Outcomes Study (MOS). Os itens da escala, originalmente em inglês, passaram por um processo de tradução e adaptação para o português, sendo avaliados em cinco etapas, revelando alto nível de confiabilidade e consistência interna. ${ }^{8,10-11} \mathrm{~A}$ escala, composta por 19 itens, contém cinco dimensões do apoio social: material (quatro perguntas); afetivo (três perguntas); emocional (quatro perguntas); informação (quatro perguntas); interação social positiva (quatro perguntas). Os escores foram obtidos por meio da soma de pontos atribuídos às perguntas de cada dimensão. A cada uma das respostas foi atribuído um escore que variou de 1 a 4: um ponto para a alternativa "nunca", dois pontos para "raramente", três pontos para "às vezes" $\mathrm{e}$ quatro pontos para "quase sempre" e "sempre" .,10-11

0 resultado da razão do total de pontos, obtidos pela pontuação máxima de cada dimensão, foi multiplicado por 100. Os índices de apoio foram considerados como variável contínua. Quanto maior o escore, maior o nível de apoio social. Utilizouse como ponto de corte os valores dos tercis a partir do escore total da escala entre os controles.

Inicialmente todas as variáveis independentes foram testadas em relação à variável dependente (prematuridade). 0 teste qui-quadrado foi utilizado para verificar diferenças entre os grupos analisados durante a análise bivariada. Foi considerado, na avaliação da significância, o valor de pd" 0,05. Nos modelos de regressão logística obteve-se a estimativa de razão de chances (OR) bruta de associação entre cada uma das variáveis independentes e a variável dependente (prematuridade) e seus intervalos de confiança (IC de 95\%). A OR é a chance de exposição no grupo que sofreu o desfecho dividida pela chance de exposição no grupo que não sofreu 0 desfecho.

Os dados coletados foram digitados para um banco de dados eletrônico, construído a partir do programa Epi Info 2008 versão 3.5.1. Para análise e processamento dos dados, foi utilizado o programa SPSS 17.

Por envolver seres humanos, o estudo foi submetido e aprovado pelo Comitê de Ética e Pesquisa da Secretaria Municipal de Saúde - CEP SMS-RJ, através do protocolo N ${ }^{\circ}$ 247/08 CAAE 0281.0.314.313-08. Antes do preenchimento do instrumento de coleta de dados, cada participante foi informada sobre os propósitos do estudo e assinou o Termo de Consentimento Livre e Esclarecido.

\section{RESULTADOS}

A população do presente estudo é formada por 108 casos e 228 controles. A idade das mulheres variou entre 14 e 45 anos. A média da idade foi de 25,8 anos (desvio-padrão: 6,9) para os casos e de 24,9 (desviopadrão 6,5) para os controles. Verifica-se nos dois grupos uma concentração de mulheres na faixa etária entre 20 e 34 anos. Das mulheres, $28,7 \%$ dos casos e $24,1 \%$ dos controles não completaram o primeiro grau (valor $p=0,51)$. Quanto à raça/etnia autorreferida, $77,8 \%$ denominaram-se não brancas no grupo de casos e $76,8 \%$ no de controles (valor $p=0,83$ ). Mulheres cuja raça/etnia autorreferida foi não branca apresentaram um risco um pouco mais acentuado para prematuridade, no entanto não se observou associação estatística.

Quanto ao trabalho remunerado, quase $60 \%$ dos casos e dos controles não desenvolviam atividade remunerada. Referiram renda familiar abaixo de um salário mínimo $21,3 \%$ dos casos e $18 \%$ dos controles (valor $p=0,74)$. Uma proporção expressiva de mulheres informou que se dirigiu a outro hospital antes de ser admitida na maternidade em que seu filho nasceu $(35,2 \%$ dos casos e $25,9 \%$ dos controles). Foi constatado que das mulheres que possuem casa própria, 80,3\% moram em aglomerados subnormais (favelas). Entre os casos $25 \%$ não tinha um companheiro, dos controles a proporção foi um pouco menor: $23,7 \%$ (valor $p=0,79$ ). Não foram encontradas diferenças significativas entre casos e controles para as variáveis: idade biológica da mãe, cor/etnia, situação conjugal, escolaridade, trabalho remunerado e renda familiar (Tabela 1).

Entre os 108 casos de nascimento prematuro, $2,1 \%$ foram classificados como prematuros extremos ( 20 a 27 semanas) e 3,3\% tinham entre 28 a 31 semanas de gestação (prematuridade moderada). Nascimentos com idade gestacional entre 32 e 36 semanas representaram $26,8 \%$ daqueles considerados próximos ao termo ou pré-termo leve (Tabela 2). A idade gestacional média para casos foi de 33,3 semanas (desvio-padrão $=3,25$ ) e para os controles, 39,2 semanas (desvio-padrão $=1,53$ ).

Entre os controles, 2,2\% não realizaram acompanhamento pré-natal, entre os casos essa proporção foi ainda maior $(7,4 \%)$ (valor $p=0,02)$. No grupo de casos, $52,8 \%$ tiveram seus filhos através de parto cesáreo, entre os controles, $31,1 \%$ (valor $p<0,001$ ). História de prematuridade anterior foi relatada por $20,4 \%$ dos casos e por $5,7 \%$ dos controles (valor $p<0,001$ ). Dos casos, $12 \%$ apresentaram gestação múltipla, entre os controles observou-se 1,8\% (valor $p<0,001$ ) (Tabela 2). 
Histórias de prematuridade anterior e gestação apresentaram os maiores valores da OR não ajustada, respectivamente $0 R=4,23$ (IC 95\% 2,03-8,77) e $\mathrm{OR}=7,66$ (IC 95\% 2,43-24,1). As mulheres submetidas à cesariana, quando comparadas àquelas que tiveram filhos através de parto natural, apresentaram um risco aumentado para prematuridade $(\mathrm{OR}=2,47$; IC $95 \%$ 1,54-3,95). Verificou-se um sobrerrisco elevado para prematuridade entre aquelas mulheres que não foram acompanhadas durante o pré-natal $(0 R=3,56$; IC $95 \%$ 1,13-11,1). 0 fumo na gravidez apontou para uma associação positiva com a prematuridade, muito embora sem significância estatística (OR=1,76, IC 95\% 0,933,33) (Tabela 2).

Todas as variáveis relacionadas à saúde e assistência quando analisadas com a variável dependente (prematuridade) apresentaram associação estatística significativa, com exceção do fumo na gravidez (Tabela 2).

Tabela 1: Características sociodemográficas das puérperas atendidas em uma maternidade da rede pública do município do Rio de Janeiro, 2009 - distribuição percentual de casos e controles, odds ratios brutos, intervalos com 95\% de confiança evalor $p$.

\begin{tabular}{|c|c|c|c|c|c|c|c|}
\hline \multirow{2}{*}{$\begin{array}{c}\text { Características } \\
\text { Sociodemográficas }\end{array}$} & \multicolumn{2}{|c|}{ Casos } & \multicolumn{2}{|c|}{ Controle } & \multirow[t]{2}{*}{$* p$} & \multirow[t]{2}{*}{ Or } & \multirow[t]{2}{*}{ IC 95\% } \\
\hline & $\bar{N}$ & $\%$ & $\bar{n}$ & $\%$ & & & \\
\hline \multicolumn{8}{|l|}{ Idade mãe } \\
\hline $14-19$ anos & 23 & 21,3 & 53 & 23,2 & 0,90 & 0,90 & $0,51-1,58$ \\
\hline $20-34$ anos & 73 & 67,6 & 152 & 66,7 & & 1 & \\
\hline 35 ou mais & 12 & 11,1 & 23 & 10,1 & & 1,08 & $0,51-2,30$ \\
\hline \multicolumn{8}{|l|}{ Cor/Etnia } \\
\hline branca & 24 & 22,2 & 53 & 23,2 & 0,83 & 1 & \\
\hline Nãobranca & 84 & 77,8 & 175 & 76,3 & & 1,06 & $0,61-1,83$ \\
\hline \multicolumn{8}{|l|}{ Situação Conjugal } \\
\hline Com companheiro & 81 & 75,0 & 174 & 76,3 & & 1 & \\
\hline Sem companheiro & 27 & 25,0 & 54 & 23,7 & 0,79 & 1,07 & $0,63-1,82$ \\
\hline \multicolumn{8}{|l|}{ Escolaridade } \\
\hline $\begin{array}{l}\text { Ensino Fundamental } \\
\text { completo/incompleto }\end{array}$ & 31 & 28,7 & 55 & 24,1 & & 0,98 & $0,42-2,27$ \\
\hline $\begin{array}{l}\text { Ensino Médio } \\
\text { completo/incompleto }\end{array}$ & 65 & 60,2 & 152 & 66,7 & 0,51 & 0,74 & $0,34-, 161$ \\
\hline Ensino Sup erior & 12 & 11,1 & 21 & 9,2 & & 1 & \\
\hline \multicolumn{8}{|l|}{ Tipo de moradia } \\
\hline Aluguel/mora de favor & 33 & 30,8 & 65 & 28,5 & 0,31 & 1,11 & $0,67-1,84$ \\
\hline Própria & 74 & 69,2 & 163 & 71,5 & & 1 & $0,66-1,69$ \\
\hline \multicolumn{8}{|l|}{ Trabalho remunerado } \\
\hline Não & 65 & 60,0 & 134 & 58,8 & 0,80 & 1,06 & $0,66-1,69$ \\
\hline $\operatorname{sim}$ & 43 & 39,8 & & 41,2 & & 1 & \\
\hline \multicolumn{8}{|l|}{ Carteira assinada } \\
\hline Não & 86 & 79,6 & 174 & 76,3 & 0,49 & 1,21 & $0,69-2,12$ \\
\hline $\operatorname{sim}$ & 22 & 20,4 & 54 & 23,7 & & 1 & \\
\hline \multicolumn{8}{|l|}{ Renda Familiar } \\
\hline Menor que 1 SM & 23 & 21,3 & 41 & 18,0 & 0,74 & 1,29 & $0,65-2,57$ \\
\hline 1 a 2 SM & 59 & 54,6 & 127 & 55,7 & & 1,07 & $0,61-1,86$ \\
\hline $3 \mathrm{SM}$ ou mais & 26 & 24,1 & 60 & 26,3 & & 1 & \\
\hline
\end{tabular}

Legenda: $\mathrm{OR}=$ odds ratio, $\mathrm{IC}=$ intervalo de confiança, ${ }^{*} P=$ teste $X^{2}$ de Pearson, $\mathrm{SM}=$ salários mínimos. 
Tabela 2-Características relacionadas à saúde e assistência das puérperas atendidas em uma maternidade da rede pública do município do Rio de Janeiro, 2009-distribuição percentual de casos e controles, odds ratios brutos, intervalos com 95\% de confiança e valor $p$.

\begin{tabular}{|c|c|c|c|c|c|c|c|}
\hline \multirow{2}{*}{$\begin{array}{l}\text { Características } \\
\text { Relacionadas à saúde } \\
\text { e assistência }\end{array}$} & \multicolumn{2}{|c|}{ Casos } & \multicolumn{2}{|c|}{ Controle } & \multirow[t]{2}{*}{$*_{p}$} & \multirow[t]{2}{*}{ Or } & \multirow{2}{*}{ IC $95 \%$} \\
\hline & $\mathrm{N}$ & $\%$ & $\mathrm{n}$ & $\%$ & & & \\
\hline \multicolumn{8}{|l|}{ Fez pré-natal } \\
\hline Sim & 8 & 7,4 & 5 & 2,2 & $0,02 *$ & 3,56 & 1,13-11,1 \\
\hline Não & 100 & 92,6 & 223 & 97,8 & & & \\
\hline \multicolumn{8}{|l|}{ Gestação } \\
\hline Múltipla & 13 & 12,0 & 4 & 1,8 & $<0,001^{*}$ & 7,66 & $2,43-24,1$ \\
\hline Única & 95 & 88,0 & 224 & 98,2 & & & \\
\hline \multicolumn{8}{|l|}{$\begin{array}{l}\text { Prematuridade } \\
\text { anterior }\end{array}$} \\
\hline Sim & 22 & 20,4 & 13 & 5,7 & $<0,001^{*}$ & 4,23 & $2,03-8,77$ \\
\hline Não & 86 & 79,6 & 215 & 94,3 & & & \\
\hline \multicolumn{8}{|l|}{ Tipo de parto } \\
\hline cesáreo & 57 & 52,8 & 71 & 31,1 & $<0,001^{*}$ & 2,47 & $1,54-3,95$ \\
\hline vaginal & 51 & 47,2 & 157 & 68,9 & & & \\
\hline \multicolumn{8}{|l|}{ Fumo na gravidez } \\
\hline Sim & 20 & 18,5 & 26 & 11,4 & 0,07 & 1,76 & $0,93-3,33$ \\
\hline Não & 88 & 81,5 & 202 & 88,6 & & & \\
\hline
\end{tabular}

Os escores calculados apresentaram distribuiç̃ẽes assimétricas com tendência a valores mais altos. As mulheres deste estudo, apesar de serem de baixa renda, apresentaram-se nos níveis mais altos do apoio. Não foi observada associação estatística entre a medida relacionada ao nível de apoio e as variáveis socioeconômicas e demográficas (Tabela 3). Entre as características relacionadas à saúde e assistência, apresentou associação estatística significativa com o apoio material apenas a história de prematuridade anterior; as demais variáveis não apresentaram valor de $p$ significativo (Tabela 4).

Tabela 3 - Características sociodemográficas das puérperas atendidas em uma maternidade da rede pública do município do Rio de Janeiro e o nível total do apoio social, 2009.

\begin{tabular}{|c|c|c|c|c|c|c|c|c|}
\hline \multicolumn{9}{|c|}{ Nível de apoio } \\
\hline \multirow[b]{2}{*}{$\begin{array}{c}\text { Características } \\
\text { Sociodemográficas }\end{array}$} & \multicolumn{4}{|c|}{ Casos } & \multicolumn{4}{|c|}{ Controle } \\
\hline & $\begin{array}{c}20 \text { a } 75 \\
\text { (baixo) } \\
\%\end{array}$ & $\begin{array}{c}76 \text { a } 87 \\
\text { (médio) } \\
\%\end{array}$ & $\begin{array}{c}88 \text { a } 100 \\
\text { (baixo) } \\
\%\end{array}$ & $* p$ & $\begin{array}{c}20 \text { a } 75 \\
\text { (baixo) } \\
\%\end{array}$ & $\begin{array}{c}76 \text { a } 87 \\
\text { (médio) } \\
\%\end{array}$ & $\begin{array}{c}88 \text { a } 100 \\
\text { (baixo) } \\
\%\end{array}$ & $* p$ \\
\hline \multicolumn{9}{|l|}{ Idade mãe } \\
\hline $14-19$ anos & 12,5 & 29,0 & 22,2 & 0,35 & 16,7 & 23,0 & 27,1 & 0,52 \\
\hline $20-34$ anos & 81,3 & 58,1 & 64,4 & & 73,3 & 63,9 & 64,5 & \\
\hline 35 ou mais & 6,3 & 12,9 & 13,3 & & 10 & 13,1 & 13,3 & \\
\hline \multicolumn{9}{|l|}{ Cor/Etnia } \\
\hline branca & 28,1 & 12,9 & 24,4 & 0,31 & 18,3 & 21,3 & 27,1 & 0,40 \\
\hline Não branca & 71,9 & 87,1 & 75,3 & & 81,7 & 78,7 & 72,9 & \\
\hline \multicolumn{9}{|l|}{ Situação Conjugal } \\
\hline Com compan heiro & 25,0 & 22,6 & 26,7 & 0,92 & 25,0 & 21,3 & 24,3 & 0,87 \\
\hline Sem companheiro & 75,0 & 77,4 & 73,3 & & 75,0 & 78,7 & 75,7 & \\
\hline \multicolumn{9}{|l|}{ Escolaridade } \\
\hline $\begin{array}{l}\text { Ensino Fundamental } \\
\text { completo/incompleto }\end{array}$ & 31,3 & 32,3 & 24,4 & 0,91 & 28,3 & 23,0 & 22,4 & 0,87 \\
\hline $\begin{array}{l}\text { Ensino Médio } \\
\text { completo/incompleto }\end{array}$ & 59,4 & 54,8 & 64,4 & & 65,0 & 67,2 & 67,3 & \\
\hline Ensino Superior & 9,4 & 12,9 & 11,1 & & 6,7 & 9,8 & 10,3 & \\
\hline \multicolumn{9}{|l|}{ Renda Familiar } \\
\hline Menor que 1 SM & 31,3 & 22,6 & 13,3 & 0,17 & 16,7 & 19,7 & 17,8 & 0,78 \\
\hline 1 a 2 SM & 56,3 & 54,8 & 53,3 & & 58,3 & 59,0 & 52,3 & \\
\hline 3 SM ou mais & 12,5 & 22,6 & 33,3 & & 25,0 & 21,3 & 29,9 & \\
\hline
\end{tabular}

Legenda: * $P=$ teste $X^{2}$ de Pearson; $S M=$ Salários MínimosTabela 4-Características relacionadas à saúdee assistência das puérperas atendidas em uma maternidade da rede pública do município do Rio de Janeiro e o nível total do apoio social, 2009. 
Tabela 4 - Características relacionadas à saúde e assistência das puérperas atendidas em uma maternidade da rede pública do município do Rio de Janeiro e o nível total do apoio social, 2009.

\begin{tabular}{|c|c|c|c|c|c|c|c|c|}
\hline \multirow{2}{*}{$\begin{array}{l}\text { Características } \\
\text { Relaciona das à saú de } \\
\text { e assistência }\end{array}$} & \multicolumn{4}{|c|}{ Casos } & \multicolumn{4}{|c|}{ Controle } \\
\hline & $\begin{array}{c}20 \text { a } 75 \\
\text { (baixo) } \\
\%\end{array}$ & $\begin{array}{c}76 \text { a } 87 \\
\text { (médio) } \\
\%\end{array}$ & $\begin{array}{c}88 \text { a } 100 \\
\text { (baixo) } \\
\%\end{array}$ & $\mathrm{P}^{*}$ & $\begin{array}{c}20 \text { a } 75 \\
\text { (baixo) } \\
\% \\
\end{array}$ & $\begin{array}{c}76 \text { a } 87 \\
\text { (baixo) } \\
\%\end{array}$ & $\begin{array}{c}88 \text { a } 100 \\
\text { (baixo) } \\
\%\end{array}$ & $\mathrm{P}^{*}$ \\
\hline \multicolumn{9}{|l|}{ Fez pré-natal } \\
\hline Sim & 12,5 & 3,2 & 6,7 & 0,36 & 3,3 & 3,3 & 0,9 & 0,41 \\
\hline Não & 87,5 & 96,8 & 93,3 & & 96,7 & 96,7 & 99,1 & \\
\hline \multicolumn{9}{|l|}{ Gestação } \\
\hline Múltipla & 12,5 & 12,9 & 11,1 & 0,96 & 1,7 & 0 & 2,8 & 0,41 \\
\hline Única & 87,5 & 87,1 & 88,3 & & 98,3 & 100 & 97,2 & \\
\hline \multicolumn{9}{|l|}{$\begin{array}{l}\text { Prematuridade } \\
\text { anterior }\end{array}$} \\
\hline Sim & 34,4 & 6,5 & 20,0 & 0,02 & 11,7 & 6,6 & 1,9 & 0,03 \\
\hline Não & 65,6 & 93,5 & 80,0 & & 88,3 & 93,4 & 98,1 & \\
\hline \multicolumn{9}{|l|}{ Tipo de parto } \\
\hline cesáreo & 59,4 & 54,8 & 46,7 & 0,52 & 28,3 & 34,4 & 30,8 & 0,76 \\
\hline vaginal & 40,6 & 45,2 & 53,3 & & 71,7 & 65,6 & 69,2 & \\
\hline \multicolumn{9}{|l|}{ Fumo na gravidez } \\
\hline Sim & 28,1 & 16,1 & 13,3 & 0,23 & 13,3 & 11,5 & 10,3 & 0,83 \\
\hline Não & 71,9 & 83,9 & 86,7 & & 86,7 & 88,5 & 89,7 & \\
\hline
\end{tabular}

Legenda: ${ }^{*} P=$ teste $X^{2}$ de Pearson.

\section{DISCUSSÃO}

Muito embora no presente estudo grande parte das características socioeconômicas e demográficas estudadas, tais como idade, escolaridade, situação conjugal, renda familiar, não tenha apresentado associação com a dimensão do apoio material e nem com o desfecho (prematuridade), o perfil socioeconômico de uma população pode ser um fator importante na determinação de alguns desfechos negativos. Acredita-se que estas variáveis têm influência marcante no acesso aos serviços de atendimento a gravidez e ao nascimento. Adicionalmente, tais fatores podem afetar de forma indireta a vida dessas mulheres, considerandose 0 acesso à rede de apoio social informal, levando ao comprometimento ou diminuição dos cuidados com a saúde durante a gestação. ${ }^{12-13}$

Em relação à idade materna, observou-se que a distribuição entre as faixas de idade da mãe eram bastante semelhantes entre casos e controles. Estudos têm mostrado que mulheres com idade inferior a 20 anos e/ou superior a 45 apresentam um maior risco para a prematuridade; a baixa escolaridade materna e a ausência de companheiro também apresentaram risco para o nascimento prematuro. ${ }^{12-14}$

Nesse estudo foi observada associação estatística significativa entre o nascimento prematuro e fatores relacionados à saúde e assistência (realização de pré-natal, tipo de gestação, prematuridade anterior, tipo de parto). A assistência prénatal permite a identificação e tratamento de inúmeras complicações durante a gestação, assim como redução ou eliminação de fatores e comportamentos de risco passíveis de correção. ${ }^{1,14}$ No Brasil, além das causas específicas relacionadas à mulher e à gestação, estão fatores relacionados ao acesso aos serviços de saúde, o que torna esse grupo ainda mais vulnerável a desfechos negativos, dentre as quais o nascimento precoce ou prematuro. ${ }^{12} 0$ aumento da cobertura pré-natal poderia, por exemplo, proporcionar resultados expressivos para a redução da prevalência de prematuridade. Uma cobertura pré-natal eficaz é capaz de promover resultados imediatos na diminuição da prematuridade, entretanto a precariedade deste atendimento encontra-se entre os responsáveis pelo aumento da prevalência do nascimento prematuro. Uma questão fundamental para 0 planejamento do setor saúde é a distribuição espacial dos serviços e da população usuária. Do ponto de vista geográfico, os serviços de saúde estão concentrados em determinados espaços ou lugares, e, consequentemente, as pessoas que se encontram mais distante são as que possuem maiores dificuldades de acesso

A gestação múltipla apresentou associação mais elevada em relação ao risco para o nascimento prematuro. Tal aspecto aumenta os riscos de complicações na gravidez, 
levando a intercorrências clínicas que vão culminar no nascimento prematuro. ${ }^{14}$ Prematuridade anterior aumenta significativamente a chance de recorrência. ${ }^{3}$ Nesse estudo, 0 tipo de par to esteve associado ao nascimento prematuro. No presente estudo constatou-se que o tipo de parto esteve associado ao nascimento prematuro. Nos últimos anos, as taxas de cesarianas têm aumentado significativamente em todo mundo, ficando muito aquém do parâmetro sugerido pala OMS (Organização mundial de saúde). Estudos brasileiros têm mostrado o parto normal associado à maior mortalidade neonatal quando comparado ao cesariano, sugerindo máqualidade da assistência ao parto vaginal, atrelado a realização de cesarianas em gestações de baixo risco e de parto vaginal nas de alto risco. ${ }^{15}$ Por outro lado, o aumento do parto cirúrgico (cesáreo) vem se tornando progressivo principalmente nas camadas socioeconômicas privilegiadas, enquanto que entre as classes menos favorecidas da população estes índices são bem menores.

A nicotina e o monóxido de carbono substâncias componentes do cigarro são vasoconstrictores e interagem em danos placentários com decréscimo no fluxo sanguíneo uterino. Tais fatores tendem a restringir o crescimento fetal, contribuindo com a ocorrência da prematuridade. ${ }^{16}$ No presente estudo, no entanto, essa associação não foi observada.

A escolha por uma população de puérperas assistidas em uma rede pública de saúde, com nível socioeconômico desfavorável, onde provavelmente o apoio social disponível fosse mais baixo, configuraria associação com o desfecho devido a sua vulnerabilidade em desenvolver o risco diante de situações adversas. No entanto, verificamos mulheres menos favorecidas com níveis/escores alto de apoio. É possível que no período gestacional a mulher tenha uma percepção mais favorável a respeito do apoio recebido em relação a aspectos emocionais e afetivos. ${ }^{9,17}$

As redes sociais apresentam expressiva importância na medida em que influenciam a autoimagem do indivíduo e são centrais para a experiência de identidade e competência, muito particularmente na atenção à saúde e adaptação em situações adversas. ${ }^{6,18}$

Uma grande variedade de fatores está presente na determinação da prematuridade; alguns possuem efeito direto, como os determinantes biológicos, outros apresentam um efeito indireto, como no caso dos determinantes socioeconômicos e psicossociais que, de alguma maneira, interferem na vida e na saúde dos indivíduos. Os dados relativos à amostra utilizada neste estudo indicam que a associação entre o apoio material e o nascimento prematuro está relacionada às condições sociais e econômicas em que vive a mulher, uma vez que o apoio material está inteiramente relacionado à provisão de recursos e de ajuda material em situações práticas da vida. Dentre as limitações desse estudo estão o tamanho amostral e a alta homogeneidade dos grupos estudados em relação às características psicossociais.
Acredita-se que a investigação do apoio social associado à prematuridade deva ser encaminhada entre grupos de maior contraste social, a fim de estudar o comportamento desta associação em outros contextos.

\section{CONCLUSÃO}

Apesar dos esforços nos últimos anos, no sentido de incorporar aspectos sociais e psicossociais aos estudos epidemiológicos, as dimensões que se referem à rede e ao apoio social ainda permanecem pouco exploradas. As características da rede social nessa pesquisa revelam que a maior fonte de ajuda e apoio é do pai do recém-nascido e da mãe da puérpera, indicando que as relações familiares constituem importante fonte de apoio, capaz de propiciar os recursos necessários ao desenvolvimento e bem-estar dos seus integrantes. Embora não tenha sido observada uma associação significativa das demais dimensões do apoio social com a prematuridade, sabe-se que o apoio representa um recurso valioso em um momento de transição existencial e grande vulnerabilidade como é o período gestacional, uma vez que pode proporcionar maior confiança e segurança para o enfrentamento de situações difíceis que podem ocorrer.

0 suporte social vem de uma ampla rede social, que inclui recursos da comunidade, parentes, amigos e instituições de saúde. Embora haja perspectivas, a médio e longo prazo, de promover a atenção humanizada neonatal em um número maior de instituições, o esforço das políticas de saúde deve ser de prevenção dos fatores de risco para a prematuridade. Assim, através de uma da qualidade da assistência prestada, os profissionais de saúde devem ser capazes de reconhecer todo o contexto social que permeia a gestante, identificando riscos biológicos e psicossociais potenciais. Tal medida favorece a prevenção do nascimento prematuro.

\section{REFERÊNCIAS}

1. Kilsztajn S, Rossbach A, Carmo MS, Sugahara GT. Prenatal care, low birth weight and prematurity in São Paulo State, Brazil, 2000. Rev Saude Publica. 2003 Jun; 37 (3): 303-10.

2. Goldenberg RL, Culhane JF, lamn JD, Romero R. Epidemiology and causes of preterm birth. Lancet. 2008 Jan; 371 (9606): 75-84.

3. Araújo BF, Tanaka ACD. Fatores de risco associados ao nascimento de recém nascidos de muito baixo peso em uma população de baixa renda. Cad Saude Publica. 2007 dez; 23 (12): 2869-77. 
4. Fischer ABA, Liao M, Mosca L. Physical activity as a potential mechanism through which social support may reduce cardiovascular disease risk. J Cardiovasc Nurs. 2008 Mar/Apr; 23(2): 90-6.

5. Cobb S. Social support as a moderator of life stresss. Psychosom Med. 1976 Sept/Oct; 38 (5): 300-13.

6. Sluzki CA. A rede social na prática sistêmica: alternativas terapêuticas. São Paulo(SP): Casa do Psicólogo; 1997.

7. Gvaz F, Karaoz S, Goz M, Ekiz S, Cetin I. Effects of the diabetic patients' perceived social support on their quality-of-life. J Clin Nurs. 2007 Jul; 16(7): 1353-360.

8. Griep RH, Chor D, Faerstein E, Lopes CS. Apoio social: confiabilidade teste-reteste de escala no Estudo Pró-Saúde. Cad Saude Publica. 2003 mar/abr; 19(2): 625-34.

9. Feldman PJ, Dunkel-Schetter C, Sandman CA, Wadhwa PD. Maternal Social Support Predicts Birth Weigth And Fetal Growth In Human Pregnancy. Psychosom Med. 2000 set/out; 62: 715-25.

10. Silva KS. Associação entre apoio social e pré-eclâmpsia: interação com paridade [tese]. Rio de Janeiro (RJ): Instituto de Medicina Social, Universidade do Estado do Rio de Janeiro; 2005.

11. Griep RH, Chor D, Faerstein E, Werneck GL, Lopes CS. Validade de constructo de escala de apoio social do Medical Outcomes Study adaptada para o português no Estudo Pró-Saúde. Cad Saude Publica. 2005 maio/ jun; 21(3): 703-14.

12. Cascaes AM, Gauche H, Baramarchi FM, Borges CM, Peres KG. Prematuridade e fatores associados ao estado de Santa Catarina, Brasil, no ano de 2005: análise dos dados do sistema de informações sobre nascidos vivos. Cad. Saude Publica. 2008 maio; 24(5): 102432.

13. Alvarado R, Medina E, Aranda W. The effect of psychosocial variables during pregnancy and in birth weight and gestacional age of the newborn. Rev Med Chile. 2002 May; 130: 561-68.

14. Ramos HAC, Cuman RKN. Fatores de risco para prematuridade: pesquisa documental. Esc Anna Nery. 2009 jun; 13 (2): 297-304.

15. Fabri RH, Silva HSL, Lima RV, Murta EFC. Estudo comparativo das indicações de cesariana entre um hospital público universitário e um hospital privado. Rev Bras Saude Mater Infant. 2002 jan/abr; 2 (1): 29-35.

16. Leopércio W, Gigliotti A. Tabagismo e suas peculiaridades durante a gestação: uma revisão crítica. J Bras Pneumol. 2004 mar/abr; 30 (2): 176-85.

17. Pinto ILG, Garcia ACO, Bocchi SCM, Carvalhaes MABL. Características do apoio social oferecido a idosos de área rural assistida pelo PSF. Cienc Saude Colet. 2006 jul/set.; 11(3): 753-64.

18. Paula ES, Nascimento LC, Rocha SMM. A influência do apoio social para o fortalecimento de famílias com crianças com insuficiência renal crônica. Rev Latino-am Enfermagem. 2008 ago; 16(4): 69299. 